\title{
DNA abandonment and the mechanisms of uniparental inheritance of mitochondria and chloroplasts
}

\author{
Arnold J. Bendich
}

(C) Springer Science+Business Media Dordrecht 2013

\begin{abstract}
For most eukaryotic organisms, the nuclear genomes of both parents are transmitted to the progeny following biparental inheritance. For mitochondria and chloroplasts, however, uniparental inheritance (UPI) is frequently observed. The maternal mode of inheritance for mitochondria in animals can be nearly absolute, suggesting an adaptive advantage for UPI. In other organisms, however, the mode of inheritance for mitochondria and chloroplasts can vary greatly even among strains of a species. Here, I review the data on the transmission of organellar DNA (orgDNA) from parent to progeny and the structure, copy number, and stability of orgDNA molecules. I propose that UPI is an incidental by-product of DNA abandonment, a process that lowers the metabolic cost of orgDNA repair.
\end{abstract}

Keywords autophagy $\cdot$ DNA damage $\cdot$ DNA repair $\cdot$ mitophagy $\cdot$ nucleoid $\cdot$ ROS

\begin{tabular}{|c|c|}
\hline Abbr & ions \\
\hline BPI & Biparental inheritance \\
\hline PCR & Polymerase chain reaction \\
\hline
\end{tabular}

Responsible Editor: Helder Maiato and Yves Barral.

A. J. Bendich $(\bowtie)$

Department of Biology, University of Washington,

Seattle, WA 98195, USA

e-mail: bendich@uw.edu
$m t^{-} \quad$ Mating type "minus"
ptDNA Plastid DNA
$\mathrm{mt}^{+} \quad$ Mating type "plus"
ROS Reactive oxygen species
mtDNA Mitochondrial DNA
UPI Uniparental inheritance
orgDNA Organellar DNA
UV Ultraviolet

\section{Introduction}

Non-Mendelian inheritance was discovered in plants more than a hundred years ago (Correns 1909; Baur 1909). Subsequent genetic and biochemical analyses revealed uniparental inheritance (UPI) in all eukaryotic lineages investigated. We learned that the basis for UPI is the failure to transmit organellar DNA (orgDNA) to the progeny. Remarkably, however, we have achieved essentially no understanding of why UPI is prevalent.

To address this deficiency, we will first consider the occurrence of UPI among diverse eukaryotes, followed by the properties of the DNA molecules that comprise the chromosomes in the organelles and the nucleus. This information will then be used to evaluate the mechanisms and utility of UPI. My thesis is that UPI is an incidental consequence of cost reduction in the maintenance of orgDNA that is highly susceptible to damage from oxidative stress. 


\section{The mode of inheritance for mitochondria and chloroplasts}

Why might we conclude that UPI of mitochondria and chloroplasts or their DNA is advantageous? One reason is that UPI is so common and widespread among animals, plants, and microbes that it seems likely to have adaptive significance (Gillham 1994). An example that may be compelling to some is that maternal inheritance of mitochondria is nearly absolute in animals. On the other hand, the mode of organellar inheritance can be highly variable in other eukaryotic lineages. For example, as described below, the pattern of inheritance for mitochondria and chloroplasts is so variable among strains of green algal species that it would be difficult to identify an advantage for either UPI or biparental inheritance (BPI).

Mammals are anisogamous (the egg is much larger than the sperm), so a bias might be associated with the unequal contribution of mitochondria to the zygote. However, UPI is also found in organisms with isogamous gametes, so that the unequal contribution of organelles alone cannot explain UPI.

Although UPI is common, the mode (UPI male or mating type "minus" $\left[\mathrm{mt}^{-}\right]$, UPI female or mating type "plus" $\left[\mathrm{mt}^{+}\right]$, or BPI) can vary greatly among strains of green (chlorophytic) algal species (Miyamura 2010). For example, in the isogamous Ulva compressa, plastid DNA (ptDNA) inheritance can be UPI $m t^{-}$, UPI $m t^{+}$, or BPI, depending on the strains crossed, and mitochondrial DNA (mtDNA) inheritance is similarly variable. In the anisogamous Caulerpa, the frequency of male gametes that have plastid nucleoids (indicating ptDNA) varies from 0.6 to $18.2 \%$ among species, and both male and female gametes always contain one to eight mitochondrial nucleoids. In Chlamydomonas reinhardtii (considered in detail in the succeeding paragraphs), the ptDNA is destroyed just after gametes fuse to form the zygote, leading to predominantly UPI $\mathrm{mt}^{+}$, whereas the mtDNA is destroyed later (at meiosis), leading to UPI $m t^{-}$. For alfalfa (Medicago sativa), plastid inheritance is paternal or biparental, with female plastids being excluded from the future embryo at the first cell division of the zygote. The proportion of progeny containing only male plastids depends on the distribution of plastids within the egg: over $90 \%$ for a weak female genotype and $41 \%$ for a strong female genotype (Mogensen 1996). For kiwifruit (Actinidia), DNA sequence analysis revealed strictly maternal inheritance of mtDNA in the progeny of all 11 crosses, whereas the ptDNA was inherited either paternally ( 9 crosses), maternally ( 1 cross), or biparentally (1 cross) (Li et al. 2013).

Such variation indicates that UPI is a quantitative trait (Birky 2008). Whereas the nearly absolute maternal inheritance of mtDNA in animals might be compatible with an advantage for UPI, the within-species variation described previously makes it difficult to identify an advantage for UPI. UPI might have little adaptive significance per se and might be a secondary consequence of other evolutionary events (Birky 1983, 2008; Xu 2005).

\section{Organellar DNA molecules: structure, copy number, maintenance, and stability}

Properties of the chromosomal DNA molecules in the nucleus and organelles are summarized in Table 1. The mitochondria of wild-type microbes and plants contain chromosomal DNA molecules in linear form, with a minor fraction of the mtDNA as circles of subgenomic size that probably originate from defective cells in the population (Bendich 2010a). In contrast, nearly all the mtDNA in most animals exists in genome-sized circles, except that most cnidarians contain linear mtDNA (Kayal et al. 2012). Although the structural data for ptDNA are largely restricted to angiosperms, the data indicate that chromosomal DNA molecules in plastids are also linear, and the genome-sized circles that represent a few percent of total ptDNA are found in cells in which ptDNA replication no longer occurs (Oldenburg and Bendich 2004a; Oldenburg et al. 2006).

Except for most animals, DNA replication may follow the recombination-dependent process used by bacteriophages T4 (for mtDNA) and T7 (for ptDNA) in which multigenomic "bushes" containing branch points represent the segregating genetic units (the chromosomes) (Fig. 1). It must be noted, however, that the data are meager even for yeasts (Bendich 2010a). In the nucleus, linear chromosomal DNA molecules end in telomeric structures that delineate a normal end from a broken end in need of repair. Thus, we expect that linear chromosomes in mitochondria and plastids must also be protected by telomeric structures. A variety of telomeric mtDNA end structures have been found among yeast species, including some that resemble nuclear telomeres (Nosek et al. 2006), 
Table 1 Properties of chromosomal DNA molecules

\begin{tabular}{lll}
\hline Characteristic & Nucleus & Mitochondria and plastids \\
\hline Molecular structure $^{\mathrm{a}}$ & Constant & Variable \\
DNA replication $^{\mathrm{b}}$ & Duplication & Amplification \\
DNA stability $^{\mathrm{c}}$ & High & Low \\
DNA damage $^{\mathrm{d}}$ & Low & High \\
Inheritance $^{\text {Biparental }}$ & Variable $^{\mathrm{e}}$ \\
\hline
\end{tabular}

${ }^{a}$ Checkpoint control ensures constancy of length, unbranched linear form, and ploidy level in the diploid nucleus at cell division, whereas orgDNA molecules can vary widely in such parameters even within a single organelle and during development and are usually of multigenomic size (Bendich 2007, 2010b; Oldenburg et al. 2013)

${ }^{\mathrm{b}}$ Mitosis ensures duplication of nuclear DNA. The high copy number of orgDNA may reflect an increased demand for organellar ribosomes that can only be satisfied by the increased ribosomal RNA gene number that results from genome amplification (Bendich 1987). For example, small colorless proplastids in the basal meristem develop into large green chloroplasts in the maize leaf, after which most ptDNA is degraded (Oldenburg and Bendich 2004a)

${ }^{\mathrm{c}}$ Resynthesis accompanying orgDNA degradation (turnover) is required during aerobic growth of unicellular eukaryotes (for example, Euglena). Degradation without resynthesis (abandonment) was found in light-grown leaves (where cells no longer divide) for ptDNA of four plants (Bendich 2010b) and mtDNA of maize (Oldenburg et al. 2013)

${ }^{\mathrm{d}}$ Bendich (2010b)

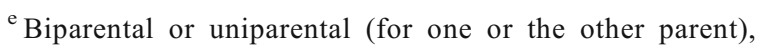
depending on the species or strain

and a 5'-protein that may serve to protect the ends of linear chromosomal DNA molecules has been reported for liverwort mitochondria (Oldenburg and Bendich 2001).

The number of copies of the genome in the nucleus of diploid organisms is two, and nuclear DNA exhibits BPI. In the organelles where UPI is common, however, genome copy number can vary from dozens to thousands. During development in diploid animals and plants, nuclear genome copy number remains at two in most somatic cells, whereas orgDNA copy number can change by 10-fold or 20-fold (Frahm et al. 2005; Oldenburg et al. 2006, 2013; Zheng et al. 2011).

DNA stability is another characteristic that distinguishes orgDNA from nuclear DNA. The most instructive example comes from the unicellular alga Euglena gracilis. Under constant growth conditions, both ptDNA and mtDNA are unstable (half-lives of 1.6 and 1.8 cell doublings, respectively), whereas nuclear DNA turnover could not be detected (Manning and Richards 1972; Richards and Ryan 1974). Other examples of orgDNA instability in animals, plants, and yeast are known (Bendich 2010b; Zheng et al. 2011; Oldenburg et al. 2013).

Most mutations are deleterious. A major source of mutation in eukaryotes is the oxidative damage to DNA that accompanies photosynthetic/respiratory electron transfer (Wright et al. 2009). For the nucleus, mutation is relatively infrequent because oxidative DNA damage is relatively minor, so that the repair of DNA damage is generally sufficient to minimize mutation. On the other hand, DNA damage is more severe in mitochondria and plastids due to on-site generation of reactive oxygen species (ROS), so that measures in addition to DNA repair are needed to minimize mutation: DNA turnover, copy number change, and abandonment. These additional measures may have led to the evolution of two major phenomena in eukaryotic biology. According to one hypothesis, embryonic development arose because of the advantage of sequestering mitochondria and plastids in metabolically "quiet" germ cells so that their DNA is not exposed to the oxidative stress produced by these organelles in "active" somatic cells (Bendich 2010b). The other (and incidental) consequence of the additional mutation-minimization measures is UPI of mtDNA and ptDNA.

\section{Minimizing mutation in organellar DNA}

Mutation caused by damage to orgDNA may be minimized at several levels:

1. DNA repair. Several repair pathways are known, including base and nucleotide excision, recombination, and strand break repair. Although DNA repair is likely to be costly in a metabolic sense, it is less costly than the subsequent levels.

2. Degradation of unrepaired orgDNA molecules. Turnover for orgDNA is high, whereas nuclear DNA is stable (Bendich 2010b).

3. Autophagy of the organelle. Defective mitochondria and plastids may be digested and their contents recycled for nutritional value (Motomura et al. 2010; Kurihara et al. 2012; Youle and van der Bliek 2012; Chan and Schon 2012).

4. Ejection of organelles or orgDNA. Regions of the cytoplasm containing defective organelles or their 


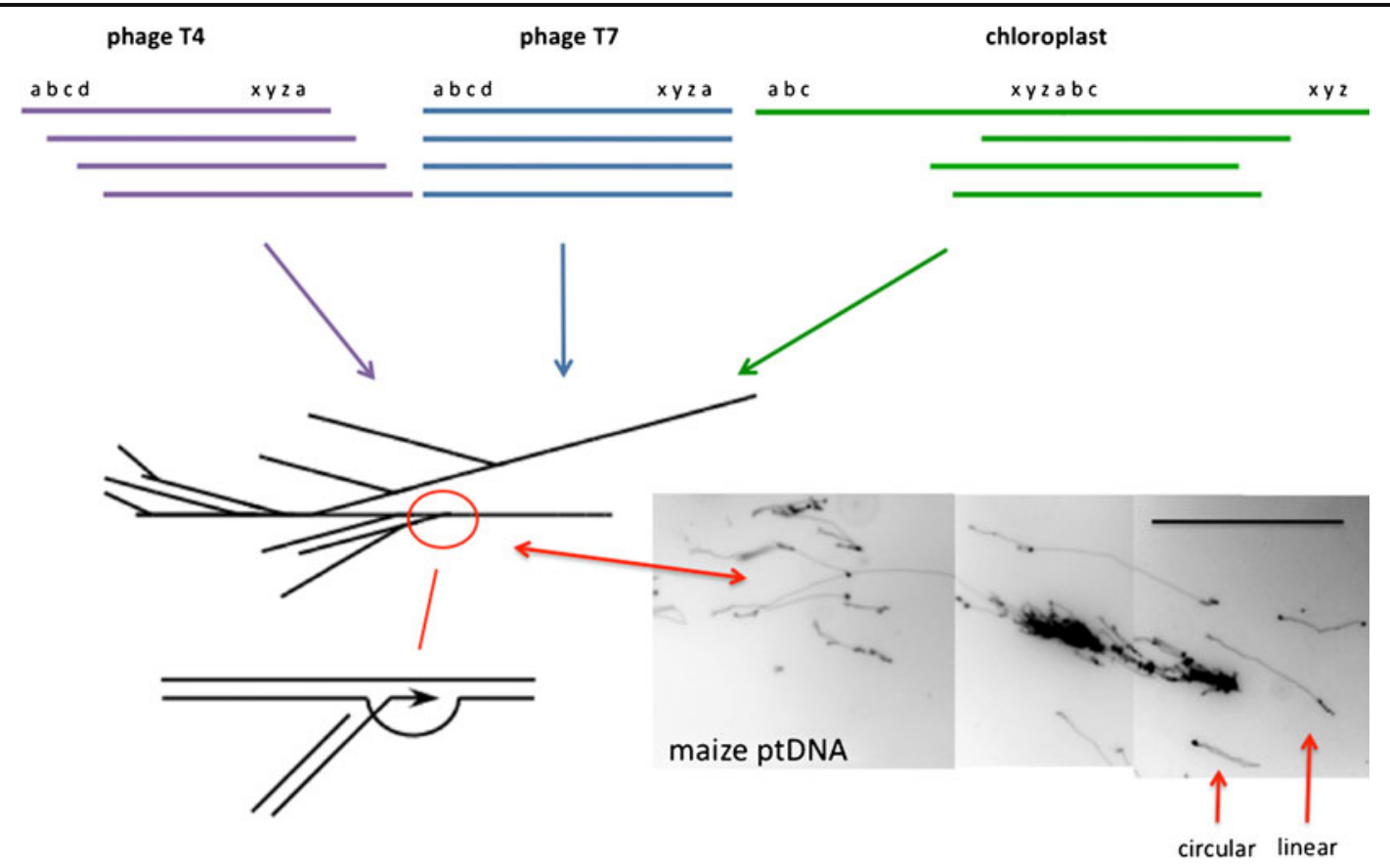

Fig. 1 Structure and replication of phage and orgDNAs. Phage T4 DNA molecules are circularly permuted and yield a circular map. T7 molecules have fixed ends. Both have repeated end sequences and use recombination-dependent replication to produce head-to-tail concatemers (not shown) and a multigenomic, branched replication intermediate. Chloroplast DNA comprises genomic concatemers (monomer, dimer, etc.) with defined end sequences. Proposed replication is T4-like for mtDNA of liverwort, S. cerevisiae, Sc. pombe, and Neurospora crassa and T7-

DNA may be extruded from the cell, thus losing their nutritive potential along with the defective orgDNA (Sears 1980; Nagata 2010; Bisharyan and Clark 2011).

5. Exclusion of organelles before, during, or just after fertilization (Sears 1980; Mogensen 1996; Nagata 2010). Although it is not known whether only damaged organelles and their DNA are thus excluded from the subsequent embryo, this is a level at which mutation in orgDNA can be minimized.

6. The last line of defense is apoptosis, whereby the entire cell is destroyed, including nuclear DNA and orgDNA (Youle and van der Bliek 2012).

\section{Genetic explanations of uniparental inheritance}

UPI might be beneficial in a "genetic" sense. Proposed genetic benefits include avoiding nuclear DNAorgDNA incompatibility, conflict between orgDNAs, like for mtDNA of Candida albicans and ptDNA, both of which end at several preferred sequences (Bendich 2010a). The ethidium-stained image shows ptDNA after maize plastids were lysed with detergent and proteinase $\mathrm{K}$. The indicated linear and circular molecules are probably genome-sized molecules of $140 \mathrm{~kb}$ (the scale bar), and the large branched molecule is probably a multigenomic chromosome during replication (Oldenburg and Bendich 2004b). Strand-displacement synthesis indicated

and the spread of selfish orgDNA in the population (Hurst et al. 1996; Burt and Trivers 2008; Birky 2008; Basse 2010; Lane 2012).

In genetic explanations of UPI, a heteroplasmic cell (one with organelles from both parents) is to be avoided. For example, mammals exhibit maternal inheritance, and the nucleus of the egg is compatible with mitochondria of the egg but not the sperm. The incompatibility is evidently due to one of the very few genes carried by mtDNA (for example, 13 protein-coding genes in mammalian mtDNA). What is not considered, however, is the vastly greater opportunity for malefemale incompatibility among the 20,000 genes contributed to the nucleus by each parent. To my knowledge, genetic explanations of genomic incompatibility do not explain how conflict is avoided in the diploid nucleus and always involve the few genes in mtDNA or ptDNA (100-200 protein-coding genes). For both mitochondria and plastids, orgDNA encodes one or a few subunits in multisubunit protein structures, including respiratory 
and photosynthetic electron-transport complexes, whereas the nucleus encodes most of those subunits (Wright et al. 2009). Nonetheless, according to the genetic benefit explanation, it is the orgDNA that is responsible for the male-female incompatibility. This omission of the seemingly overwhelming male-female opportunity for incompatibility in the nucleus is the strongest argument against the notion that the benefits of UPI are genetic.

The spread of selfish DNA elements through a sexually-reproducing population can be deleterious, so that the asexual nature of organelles with UPI prevents dissemination of such selfish DNA in the population. This is a proposed genetic benefit of UPI. There is apparent benefit from maintaining both male and female genomes in the nucleus, which contains large amounts of (originally selfish) transposon DNA, so there is no a priori reason to expect conflict among organellar genomes largely or entirely devoid of transposons. UPI is nearly absolute for animal mitochondria that do not contain apparent selfish DNA elements. I now turn to biochemical explanations of UPI for which no genomic conflict is required.

\section{Biochemical explanations for uniparental inheritance}

The first proposed "biochemical" benefit was nutritional: the recycling of superfluous ptDNA nucleotides (Sears and VanWinkle-Swift 1994). Here, I propose that a reduction in the biochemical "cost" of repairing highly-damaged orgDNA leads to UPI as an unintended and incidental consequence and that there is no genetic benefit for UPI. Whereas orgDNA structure, copy number, replication/repair, and stability are not important for genetic explanations, they are crucial characteristics in biochemical explanations.

Why maintain DNA in the organelles?

Although the metabolic cost of repairing damaged DNA has not been quantified, it is assumed to be sufficiently high that reducing such cost can profoundly affect evolutionary outcomes (Wright et al. 2009; Bendich 2010b). Since orgDNA is more susceptible to damage than is nuclear DNA and nearly all of the genes from the primordial endosymbionts have already been moved to the nucleus, why has orgDNA been retained?
Although this question has been considered for decades, the reason I find most compelling is that high vulnerability to damage allows orgDNAs to serve as sensors of redox damage and bioenergetics quality control (Wright et al. 2009).

If a mammalian mtDNA molecule is damaged but not repaired, the DNA molecule carrying the damage will be degraded in order to prevent mutagenesis (the molecule is abandoned). In a proposed mechanism, DNA or RNA polymerase is stalled at the point of damage (double-strand break (DSB), single-strand break leading to a DSB, pyrimidine dimer, and bulky DNA adduct) and provides signals for degradation (Shokolenko et al. 2009; Liu and Demple 2010).

Inheritance of organellar DNA monitored by cytological procedures

For three decades, the work of Tsuneyoshi Kuroiwa and his associates has provided us with spectacular visual images of orgDNA within cells before, during, and after the gametes unite to form the zygote. The DNA fluorophores DAPI and SYBR Green I have been used to identify and quantify the mass of orgDNA present in nucleoids. Nested PCR analysis of individual zygotes of $C$. reinhardtii shows that changes in DNA fluorescence accurately reflect changes in ptDNA mass, rather than a staining artifact (Nishimura 2010). Analogous data for individual sperm during spermatogenesis and just after fertilization in the fish Oryzias latipes lead to the same conclusion for mtDNA (Nishimura 2010). However, neither the molecular weight nor structure of the DNA molecules is revealed in these cytological data; those characteristics currently require analysis of the orgDNA after extraction from the cell.

The most instructive example of orgDNA loss in angiosperms is found during the maturation of (nonphotosynthetic) pollen (Nagata et al. 1999). The elimination of ptDNA and mtDNA from the generative cell of the pollen grain correlates perfectly with the uniparental or biparental mode of inheritance for eight species. However, both ptDNA and mtDNA are always degraded during development of the vegetative cell (which does not contribute to the zygote) of the pollen grain regardless of the organellar inheritance pattern. Thus, the demise of orgDNA can be independent of its effect on the mode of organellar inheritance. These data suggest that UPI is an unintended and 
incidental consequence of the demise of orgDNA, which provides some biochemical benefit. Such a benefit might be nucleotide recycling for nutritional purposes (Sears and VanWinkle-Swift 1994) or reducing the burden of repairing many damaged copies of orgDNA that are no longer needed for their coding function (Oldenburg and Bendich 2004a).

C. reinhardtii has long been used to study UPI (Sager 1954), and it remains the primary organism for mechanistic studies. When vegetative cells are deprived of nitrogen, they differentiate into gametes of opposite mating type. The $m t^{+}$and $m t^{-}$gametes fuse to form a zygote containing one chloroplast from each parent that carries $\sim 80-100$ genome equivalents of ptDNA distributed unequally among 5-10 nucleoids. Within $50 \mathrm{~min}$ and before the two chloroplasts fuse, the DNA-fluorescent nucleoids in the $m t^{-}$plastid usually disappear, whereas the nucleoids in the $m t^{+}$ parent persist and provide the subsequent zygospores with ptDNA (Fig. 2). The result is UPI $m t^{+}$for about $95 \%$ of the zygotes and BPI (with some UPI $m t^{-}$) for the other $5 \%$ ("exceptional zygotes").

Through the decades, the accepted explanation for UPI has been that some "protective" agent prevents a nuclease from attacking the ptDNA in the $m t^{+}$chloroplast. Protection has been attributed to selective methylation of $m t^{+}$ptDNA (based on a restriction-modification model in bacteria), blocking a $\mathrm{Ca}^{++}$-dependent nuclease from reaching the ptDNA within the $m t^{+}$chloroplast, or deprivation of $\mathrm{Ca}^{++}$from the $\mathrm{mt}^{+}$chloroplast. Nonetheless, our understanding of the molecular mechanisms is at a very preliminary stage (Nishimura 2010). One observation that predates the protection hypothesis should be recounted (Sager and Ramanis 1967). Brief UV irradiation of $m t^{+}$gametes just prior to mating prevented the selective elimination of $m t^{-}$ ptDNA in zygotes and increased the frequency of BPI. The same treatment of $m t^{-}$gametes had no effect on the transmission of ptDNA. But if the UV-irradiated $\mathrm{mt}^{+}$gametes were allowed to recover in light prior to mating, the frequency of exceptional zygotes decreased. Sager and Ramanis hypothesized that UV irradiation blocked the synthesis of or inactivated a $m t^{+}$gamete-specific gene product necessary for the elimination of $m t^{-}$cpDNA in zygotes. As an alternative, I suggest that UV irradiation damaged the $m t^{+}$ptDNA so that, unless it was repaired during the recovery period, it was
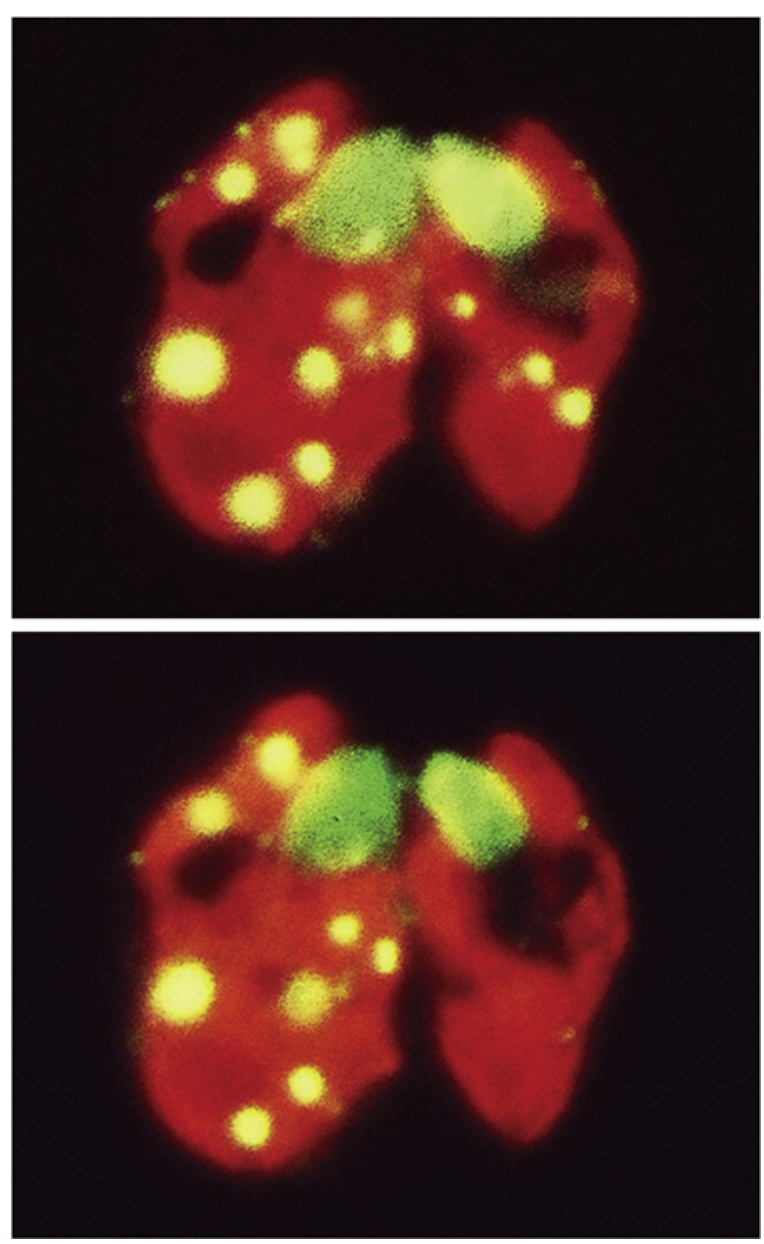

Fig. 2 Selective degradation of ptDNA in Chlamydomonas. Fluorescence microscopy of a zygote stained with SYBR Green I. Chlorophyll autofluoresces red, and stained DNA is yellow. The original $m t^{+}$and $m t^{-}$cells form the left and right halves of the zygote, respectively. Top before selective degradation, ptDNA nucleoids are seen in the unfused chloroplasts. Bottom $10 \mathrm{~min}$ later, the ptDNA in the $m t^{-}$chloroplast has been degraded, whereas DNA persists in both nuclei (the large yellow-green bodies). Photographs courtesy of Yoshiki Nishimura (Birky 2008)

degraded in the zygote. For the $m t^{-}$gametes, however, ptDNA repair had been turned off during gametogenesis so that $m t^{-}$ptDNA damaged by oxidative stress from photosynthetic electron transport remained damaged during fertilization. Once within the zygote, damaged ptDNA was degraded in order to avoid mutation in the subsequent zygospores. Thus, there is no selective "protection" of any ptDNA, only destruction of damaged ptDNA regardless of mating type origin. 


\section{To repair or not to repair?}

That is the question. The answer determines the uniparental or biparental mode of organellar inheritance.

\section{DNA abandonment}

I introduce the concept of "abandonment," whereby a damaged DNA molecule is not repaired. A damagedbut-unrepaired molecule is typically degraded in bacteria (Skarstad and Boye 1993), human mitochondria (Shokolenko et al. 2009), and mitochondria and chloroplasts of E. gracilis and maize leaves (Zheng et al. 2011; Oldenburg et al. 2013). Nuclear DNA can be abandoned during apoptosis when the entire cell is abandoned. Otherwise, the cell cycle checkpoint apparatus ensures that nuclear DNA molecules are maintained in good repair so they can act as chromosomes in inheritance (Bendich 2007). Abandonment of ptDNA in C. reinhardtii depends on the genotype of the nucleus and results in UPI $\mathrm{mt}^{+}$, whereas for mtDNA it results in UPI $m \bar{t}^{-}$.

Other than apoptosis, there is only one way to minimize mutation in the nucleus with its relatively low level of DNA damage, and that is to repair the damage. But DNA degradation and organelle elimination are alternatives to DNA repair for the high-copy organellar genomes carrying high levels of damage. The choice among alternatives will vary among organisms and will coincidentally determine the mode of organellar inheritance. I now consider several examples.

Isogamous yeasts and algae

For both Saccharomyces cerevisiae and Schizosaccharomyces pombe, BPI of mtDNA is the result if one were to analyze the progeny from a cross without first isolating the individual zygotes (Fig. 3) (Birky 2008). The zygotes contain mtDNAs from both parents (and are thus heteroplasmic), but during the ensuing cell divisions, all cells in the zygote clones become largely or completely homoplasmic. The result is UPI that is not correlated with mating type and is attributed to "a combination of factors: random partitioning of mitochondria to buds; random replication of mtDNA; and turnover of mtDNA molecules" (Birky 2008). We can modify this interpretation by specifying that most molecules are damaged and degraded, and only the few molecules that are repaired can participate in DNA replication. The molecules that are repaired are not correlated with mating type. The result is a few multigenomic bushes (chromosomes) that segregate in a few cell divisions to produce homoplasmic cells of either mating type. Thus, the cost saving in DNA repair, made possible by abandoning damaged mtDNA molecules, incidentally leads to UPI in yeast, but the mode of inheritance per se is of no adaptive significance. For Chlamydomonas, the mtDNA and ptDNA molecules that are repaired are correlated with $m t^{-}$and $m t^{+}$mating types, respectively, with a nearly $50 \%$ reduction in DNA repair cost by abandoning damaged DNA molecules. The exceptional biparental zygotes would represent a slight inefficiency in cost saving, according to this interpretation.

The apparent instability of yeast mtDNA and Chlamydomonas ptDNA in ${ }^{15} \mathrm{~N}-{ }^{14} \mathrm{~N}$ density transfer experiments ("dispersive" DNA replication) can be attributed to either high-frequency recombination or DNA turnover (Williamson and Fennell 1974; Turmel et al. 1981), but nucleotide pool effects confound interpretations. Since rapid turnover has been demonstrated for E. gracilis mtDNA and ptDNA and mung bean mtDNA (Dai et al. 2005), we may interpret the yeast mtDNA data as turnover. The half-life of mtDNA in yeast is $\sim 4 \mathrm{~h}$ (Zhang et al. 2007). For Chlamydomonas, the repair/no-repair decision is made during gametogenesis, which could explain why UPI is correlated with mating type. If that decision were made in the zygote for yeast, UPI would not be correlated with mating type.

Animals and plants

In animals, the large egg contains far more mtDNA than does the small sperm, so that maternal inheritance would be assured on statistical grounds alone. Nonetheless, sperm mtDNA is actively destroyed and does not persist in the zygote of diverse animals (Nishimura 2010; Chan and Schon 2012). Sperm motility depends on respiration, leading to ROS and mtDNA damage. The abandoned sperm mtDNA would be degraded by default, rather than selection to avoid heteroplasmy in the zygote. For a hundred years, microscopic observation has revealed numerous examples among plants and algae of the elimination of plastids and/or mitochondria during spermatogenesis 


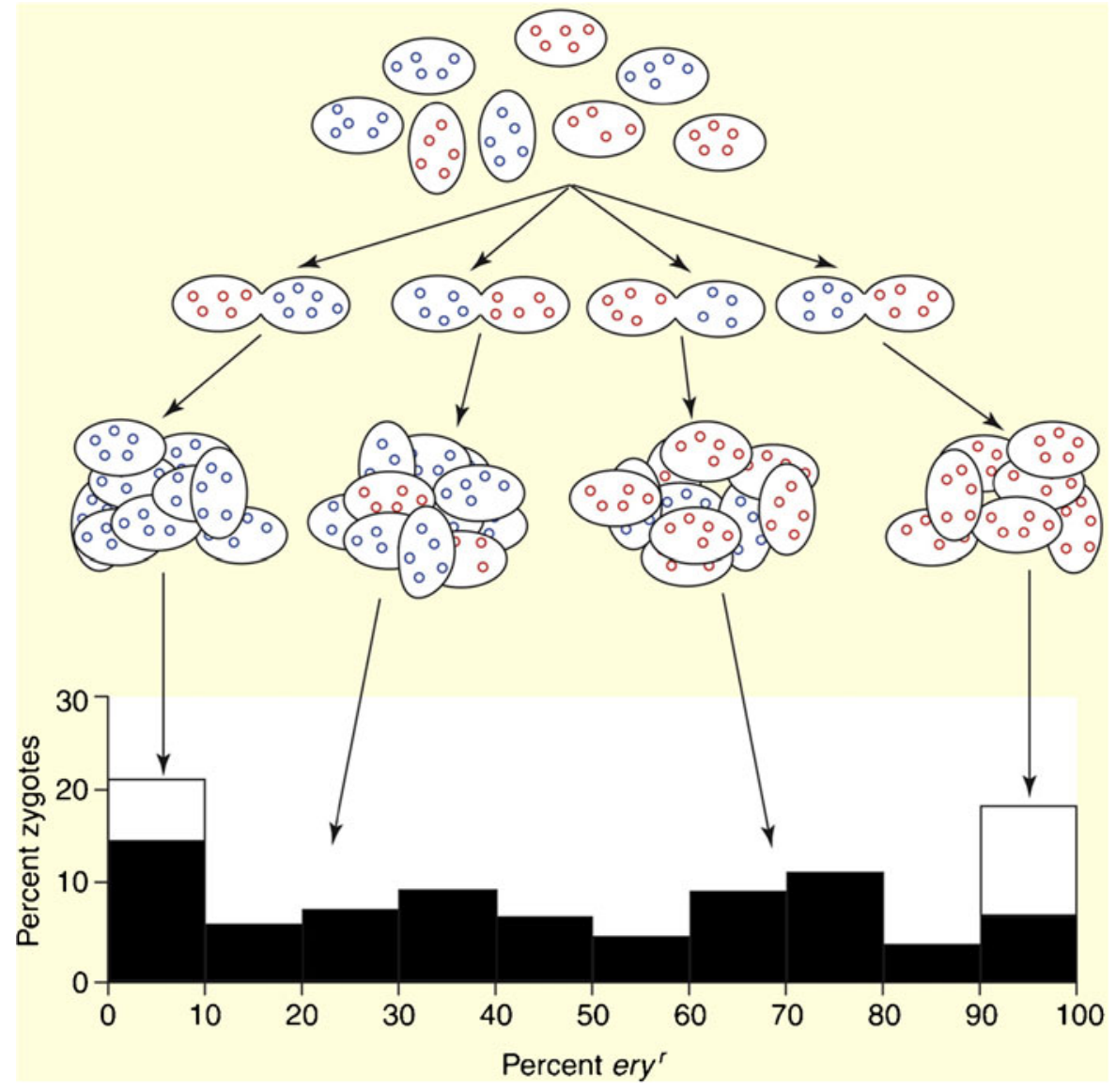

Fig. 3 Illustration of a yeast cross between strains with erythromycin-resistant $\left(\right.$ ery ${ }^{r}$; red $)$ and erythromycin-sensitive $\left(e r y^{s} ;\right.$ blue $)$ mitochondrial genomes. Haploid cells of the two genotypes are mixed (top) and fuse in pairs to form zygotes, all of which are heteroplasmic (have two alleles of a mitochondrial gene). Individual zygotes are isolated and allowed to produce zygote clones in which every cell is nearly or completely homoplasmic resistant or sensitive. (Real zygote clones contain $\geq 1,000$ cells.) Illustrated are uniparental zygote clones with 0 or $100 \%$ ery ${ }^{r}$ cells and genomes, as well as biparental zygote clones with 20 and $70 \%$ resistant genomes. At the bottom is a distribution of the mitochondrial gene frequencies in zygote clones. Note that if one analyzed a sample of progeny from

and fertilization that correlate with the mode of cytoplasmic inheritance (Sears 1980; Mogensen 1996). The organelles are eliminated by ejection from motile sperm (for example, Sphagnum moss and the fern Marsilea vestita), exclusion from the zygote, or "degeneration" (autophagy in modern parlance) within the zygote. Such elimination may remove defective organelles that would produce excessive amounts of ROS, one consequence of which is additional damage to orgDNAs. the cross without first isolating zygotes, one would not detect uniparental zygotes. Figure and revised legend from Birky (2008). Although the mitochondrial genomes are depicted as circles, the multiply-branched forms in Fig. 1 would better represent the chromosomes. To perform a yeast cross, vegetative cells are deprived of nitrogen, leading to meiosis and gametes. In $S$. cerevisiae, nitrogen deprivation also leads to increased oxidative stress and mutation in mtDNA (Kurihara et al. 2012). The indicated results would be obtained if all the mtDNA in each zygote were damaged, some mtDNA was abandoned, and only some molecules were chosen at random for repair and replication. The cells would thus become homoplasmic after one or a few cell divisions

Abandonment of orgDNA in unicellular eukaryotes may coincidentally influence UPI and BPI. For plants, orgDNA repair cost saving may be realized in two ways. First, the orgDNA damage load can be greatly reduced by not conducting respiration or photosynthesis in the germ cell lineage (Bendich 2010b). Second, for leaves of maize, there is a large lightinduced decline in the copy number and molecular integrity of orgDNA molecules relative to the stalk (Oldenburg and Bendich 2004a; Zheng et al. 2011; 
Oldenburg et al. 2013). Thus, it appears that abandonment of orgDNA may be beneficial in a biochemical sense for somatic cells not involved in sexual reproduction.

\section{Conclusions}

Frequently proposed evolutionary advantages of UPI have been based on genetic data and population theory and specify an avoidance of genomic conflict. Such proposals fail to explain why such conflict does not prevent diploidy in the nucleus and seem to be contradicted by the variability of within-species inheritance patterns. An alternative view is based on the instability and repair of damaged orgDNA molecules: there is no adaptive significance of UPI. Instead, the process by which the cost of repairing orgDNA can be lowered has led to UPI as an incidental by-product that has interested geneticists for decades.

Acknowledgments I thank George L.G. Miklos for the critical analysis during the preparation of this article.

\section{References}

Basse CW (2010) Mitochondrial inheritance in fungi. Curr Opin Microbiol 13:712-719

Baur E (1909) Das wesen und die erblichkeitsverhaltniss der "varietates albomarginatae hort" von Pelargonium zonale. Z Vererbungs 1:330-351

Bendich AJ (1987) Why do chloroplasts and mitochondria contain so many copies of their genome? Bioessays 6:279-282

Bendich AJ (2007) The size and form of chromosomes are constant in the nucleus, but highly variable in bacteria, mitochondria and chloroplasts. Bioessays 29:474483

Bendich AJ (2010a) The end of the circle for yeast mitochondrial DNA. Mol Cell 39:831-832

Bendich AJ (2010b) Mitochondrial DNA, chloroplast DNA and the origins of development in eukaryotic organisms. Biol Direct 5:42

Birky CW Jr (1983) Relaxed cellular controls and organelle heredity. Science 222:468-475

Birky CW Jr (2008) Uniparental inheritance of organelle genes. Curr Biol 18:R692-R695

Bisharyan Y, Clark TG (2011) Calcium-dependent mitochondrial extrusion in ciliated protozoa. Mitochondrion 11:909-918

Burt A, Trivers R (2008) Genes in conflict: the biology of selfish genetic elements. Belknap Press of Harvard University Press, Cambridge
Chan DC, Schon EA (2012) Eliminating mitochondrial DNA from sperm. Dev Cell 22:469-470

Correns C (1909) Verebungsversuche mit blass (gelb) grunen und buntblattrigen sippen bei Mirabilis jalapa, Urtica pilulifera und Lunaria annua. Z Vererbungs 1:291-329

Dai H, Lo YS, Litvinchuk A et al (2005) Structural and functional characterizations of mung bean mitochondrial nucleoids. Nucleic Acids Res 33:4725-4739

Frahm T, Mohamed SA, Bruse P, Gemünd C, Oehmichen M, Meissner C (2005) Lack of age-related increase of mitochondrial DNA amount in brain, skeletal muscle and human heart. Mech Ageing Dev 126:1192-1200

Gillham NW (1994) Organelle genes and genomes. Oxford University Press, New York

Hurst LD, Atlan A, Bengtsson BO (1996) Genetic conflicts. Q Rev Biol 71:317-364

Kayal E, Bentlage B, Collins AG, Kayal M, Pirro S, Lavrov DV (2012) Evolution of linear mitochondrial genomes in medusozoan cnidarians. Genome Biol Evol 4:1-12

Kurihara Y, Kanki T, Aoki Y et al (2012) Mitophagy plays an essential role in reducing mitochondrial production of reactive oxygen species and mutation of mitochondrial DNA by maintaining mitochondrial quantity and quality in yeast. J Biol Chem 287:3265-3272

Lane N (2012) The problem with mixing mitochondria. Cell 151:246-248

Li D, Qi X, Li X, Li L, Zhong C, Huang H (2013) Maternal inheritance of mitochondrial genomes and complex inheritance of chloroplast genomes in Actinidia Lind.: evidences from interspecific crosses. Mol Genet Genomics. doi:10.1007/s00438-012-0732-6

Liu P, Demple B (2010) DNA repair in mammalian mitochondria: much more than we thought? Environ Mol Mutagen 51:417-426

Manning JE, Richards OC (1972) Synthesis and turnover of Euglena gracilis nuclear and chlorplast deoxyribonucleic acid. Biochemistry 11:2036-2043

Miyamura S (2010) Cytoplasmic inheritance in green algae: patterns, mechanisms and relation to sex type. J Plant Res 123:171-184

Mogensen HL (1996) The hows and whys of cytoplasmic inheritance in seed plants. Am J Bot 383-404

Motomura T, Nagasato C, Kimura K (2010) Cytoplasmic inheritance of organelles in brown algae. J Plant Res 123:185192

Nagata N (2010) Mechanisms for independent cytoplasmic inheritance of mitochondria and plastids in angiosperms. J Plant Res 123:193-199

Nagata N, Saito C, Sakai A, Kuroiwa H, Kuroiwa T (1999) The selective increase or decrease of organellar DNA in generative cells just after pollen mitosis one controls cytoplasmic inheritance. Planta 209:53-65

Nishimura Y (2010) Uniparental inheritance of cpDNA and the genetic control of sexual differentiation in Chlamydomonas reinhardtii. J Plant Res 123:149-162

Nosek J, Kosa P, Tomaska L (2006) On the origin of telomeres: a glimpse at the pre-telomerase world. Bioessays 28:182190

Oldenburg DJ, Bendich AJ (2001) Mitochondrial DNA from the liverwort Marchantia polymorpha: circularly permuted 
linear molecules, head-to-tail concatemers, and a $5^{\prime}$ protein. J Mol Biol 310:549-562

Oldenburg DJ, Bendich AJ (2004a) Changes in the structure of DNA molecules and the amount of DNA per plastid during chloroplast development in maize. J Mol Biol 344:13111330

Oldenburg DJ, Bendich AJ (2004b) Most chloroplast DNA of maize seedlings in linear molecules with defined ends and branched forms. J Mol Biol 335:953-970

Oldenburg DJ, Rowan BA, Zhao L, Walcher CL, Schleh M, Bendich AJ (2006) Loss or retention of chloroplast DNA in maize seedlings is affected by both light and genotype. Planta 225:41-55

Oldenburg DJ, Kumar RA, Bendich AJ (2013) The amount and integrity of mtDNA in maize decline with development. Planta 237:603-617

Richards OC, Ryan RS (1974) Synthesis and turnover of Euglena gracilis mitochondrial DNA. J Mol Biol 82:57-75

Sager R (1954) Mendelian and non-Mendelian inheritance of streptomycin resistance in Chlamydomonas reinhardi. Proc Natl Acad Sci U S A 40:356-363

Sager R, Ramanis Z (1967) Biparental inheritance of nonchromosomal genes induced by ultraviolet irradiation. Proc Natl Acad Sci U S A 58:931-937

Sears BB (1980) Elimination of plastids during spermatogenesis and fertilization in the plant kingdom. Plasmid 4:233-255

Sears BB, VanWinkle-Swift K (1994) The salvage/turnover/ repair (STOR) model for uniparental inheritance in
Chlamydomonas: DNA as a source of sustenance. J Hered 85:366-376

Shokolenko I, Venediktova N, Bochkareva A, Wilson GL, Alexeyev MF (2009) Oxidative stress induces degradation of mitochondrial DNA. Nucleic Acids Res 37:2539-2548

Skarstad K, Boye E (1993) Degradation of individual chromosomes in recA mutants of Escherichia coli. J Bacteriol 175:5505-5509

Turmel M, Lemieux C, Lee RW (1981) Dispersive labelling of Chlamydomonas chloroplast DNA in ${ }^{15} \mathrm{~N}-{ }^{14} \mathrm{~N}$ density transfer experiments. Curr Genet 4:91-97

Williamson D, Fennell D (1974) Apparent dispersive replication of yeast mitochondrial DNA as revealed by density labelling experiments. Mol Gen Genet MGG 131:193-207

Wright AF, Murphy MP, Turnbull DM (2009) Do organellar genomes function as long-term redox damage sensors? Trends Genet 25:253-261

$\mathrm{Xu} \mathrm{J}$ (2005) The inheritance of organelle genes and genomes: patterns and mechanisms. Genome 48:951-958

Youle RJ, Van Der Bliek AM (2012) Mitochondrial fission, fusion, and stress. Science 337:1062-1065

Zhang Y, Qi H, Taylor R, Xu W, Liu LF, Jin S (2007) The role of autophagy in mitochondria maintenance: characterization of mitochondrial functions in autophagy-deficient $S$. cerevisiae strains. Autophagy 3:337-346

Zheng Q, Oldenburg DJ, Bendich AJ (2011) Independent effects of leaf growth and light on the development of the plastid and its DNA content in Zea species. J Exp Bot 62:27152730 